Die Rechnung ist nach der Formel $\mathrm{C}^{18} \mathrm{H}^{12} \mathrm{O}^{9}$, welche das Atomengewicht $=2350,7^{5}$ giebt.

Die Analyse der freien Säure ergab:

$$
\begin{aligned}
& \text { Kohlenstoff . . . } 61,89 \\
& \text { Wasserstoff } \cdot \text {. . 4,21 } \\
& \text { Sauerstoff }: \quad \cdot \quad 33,90
\end{aligned}
$$

Dieses stimint mit der Zusammensetzung der wasserhaltigen Japonsäure überein, und beweist, dass die Rubinsäure für sich, im ungebundenen Zuslande, nicht bestehen kann, sondern auf Kosten der Luft in dieselbe Säure übergeht, welche die Katechusäure bei Behandlung mit ätzendem Kali an der Luft und in der Wärme liefert.

Durch Behandeln mit schwachem Königswasser geht die Katechusäure in ein rostgelbes Pulver über, was ein noch näher zu untersuchender, von den angeführten aber verschiedener Stoff ist.

Wird die Katechusäure ferner in vielem Wasser gelöst, so dass sich beim Erkalten nichts ausscheidet, bleibt die Lösung mehre Wochen lang an der Luft stehen, und wird endlich in der Wärme verdunstet, so erhält man eine trockne rothe extractartige Masse, die in Wasser leichullöslich ist, also nicht Rubinsäure seyn kann, und ebenfalls noch näher zu untersuchen ist.

\title{
Ueber die Aetherarten mit organischen nicht flüchtigen Säuren;
}

$$
\text { Guerin-Varry. }
$$

Annales de Chim. et de Phys. LXII. 55.

Die Aether mit unorganischen und organischen flüchtigen Arch. d..Pharn. II. Reihe. IX. Bds. 3.Hft. 
Säuren sind der Gegenstand tiefer Studien gewesen und jetzt sehr bekannt, nicbt so ist es mit den Aetheren mit nicht flüchtigen organischen Säuren, wie die Geschichte derselben zeigt.

Th e n a rd fand bei Behandeln von Alkohol mit Citronsäure und Apfelsäure, durch Hülfe von Schwefelsäure, dass mit jeder dieser Säuren eine ölartige, geruchlose Materie entstand, schwerer als Wasser, schwerlöslich in demselben, leichtlöslich in Alkohol, und durch Alkalien in Alkohol und Citronsäure oder Apfelsäure zersetzbar, und bei der Operation nicht sich verflüchtigend. Weinsteinsäure auf dieselbe Weise behandelt, lieferte eine Flïssigkeit, woraus sich durch Wasser keine ölartige Flüssigkeit abscheiden liess. Er betrachtet das letzte Produkt als ein Gemenge von Sulfovinat und Tartrovinat von Kali, oder des ersien Salzes und Weinsteinäther.

Plisson und Henry erbielten durch Behandeln von Alkohol mit Chinasäure eine feste Zusammensetzung, die sie als Chinaäther betrachten, der nach ihnen viel Analogie mit dem Weinsteinäther Thenard's hat.

Pelouze erhielt durch Erhitzen eines Gemenges von Citronsäure, Alkohol, Salzsäure und Aether zwischen 5o bis $60^{\circ} \mathrm{C}$. Citronäther. Es scheint aber, als wenn die Aetherbildung nicht immer statt findet. Malag utti hat kürzlich Schleimsäureäther bereitet im krystallinischen Zustande, indem er Alkohol und in Schwefelsäure aufgelöste Schleimsäure einer mässigen Temperatur aussetzte.

Man sieht hieraus, dass unsere Kenntnisse über die Aetherarten mit organischen nicht flüchtigen Säuren noch sehr beschränkt sind. Ich habe mích bemüht, zur Ausfiillung dieser Lücken etwas beizutragen. Meine Arbeit besteht aus zwei Theilen. Der erste handelt über die Wirkung der Weinstein - und Paraweinsteinsäure auf den Alkohol, der andere von der Wirkung derselben Säure auf den Holzgeisı. 


\section{The il.}

\section{o. 1 . \\ Wirkung der Weinsteinsäure auf den Alkohol.}

Trommsdorff fand vor geraumer Zeit, dass die Weinsteinsäure aus einer heissen Aufösung in absolutem Alkohol beim Einengen und Erkalten nicht wieder krystallisire. Die mit Kreide behandelte Flïssigkeit gab ihm weinsieinsauren Kalk, und eine Flüssigkeit, die Weinsteinsäure, Alkohol und Kalk enthielt.

Dieser Versuch und die ätherbildende Wirkung der Oxalsäure auf Alkohol, ohne Zwischenkunft von Schwefelsäure, führten mich auf die Vermuthung, dass ich Weinsteinäther oder Tartroweinsäure, ohne eine unorganische Säure anzuwenden, würde erhalten können.

Bei mässigem Kochen löste ich ein Theil Weinsteinsäure in ihrem gleichen Gewichte absoluten Alkohol bei mässiger Wärme in einem Destillirapparate auf, erhielt die Auflösung sechs Stunden lang in einer Temperatur von 65 bis $70^{\circ}$, und theilte die syrupsartige Masse dann in zwei Theile. Den einen Theil bebandelte ich mit vielem Wasser, in der Hoffnung, Weinsteinäther zu haben, erhielt aber keine genügenden Resultate. Der andere Theil wurde mit seinem vierfachen Volum Wasser vermischt und mit kohlensaurem Baryt gesättigt. Es entstand weinsteinsaurer Baryt, der abfiltrirt wurde; die Flüssigkeit wurde bei 40 und $50^{\circ}$ eingeengt, es schied sich noch etwas weinsteinsaurer Baryt ab, worauf sie dem freiwilligen Verdumsten überlassen wurde. Sie lieferte schöne Krystalle von tartroweinsaurem Baryt. Die Erhifzung der alkoholischen Solution der Weirsteinsäure liess man sechs Stunden lang währen, in der Absicht, dass sich viel Tartroweinsäure bilden sollte, aber die zur Auflösung 
der Weinsleinsäure in kochendem Alkohol nöthige Zeit ist hinreichend, um Tartroweinsäure zu bilden.

Die Leichtigkeit, mit welcher die Weinsteinsäure sich in Tartroweinsäure verwandelt, liess mich vermuthen, dass die Bildung derselben schon bei gewöhnlicher Temp. vor sich gehen möchte, was auch der Versuch bestätigte.

Die vorstehenden Thatsachen beweisen, dass die Tartroweinsäure eine ätherbildende Kraft besitzt; die von der der Schwefelsäure nicht sehr verschieden seyn kann; denn nach Magnus bildet sich die Schwefelsäure bei gewöhnlicher Temp. nur durch Eirı wirkung concentrirter Schwefelsäure auf Alkohol, und letzter muss absolut seyn, will man viel erhalten.

Bemerkungen. Wenn man Alkohol mit Weinsteinsäure sättigt, bei einer Wärme selbst unter $100^{\circ} \mathrm{C}$., so erhält man stets eine gelblich gefärbte syrupsartige Flüssigkeit, nimmt man die Sältigung in der Kälte vor, so ist die Auflösung nie gefärbt.

Da die Gegenwart einer energischen Base oder ihres Carbonats in einigen Fällen die Bildung einer Säure in einer Verbindung veranlassen kann, in welcher solche zuvor nicht vorhanden war, so wollte ich mich überzeugen, ob die Tartroweinsäure in der alkoholischen Solution als gebildet vorhanden sey. Einen Theil reine Weinsteinsäure löste ich in einem Theil absoluten Alkohol durch leichtes Kochen auf, die Auflösung wurde $60^{\circ}$ bis $70^{\circ}$ ausgesetzt, bis $\frac{2}{3}$ der Flüssigkeit überdestillirt waren. Der syrupsartige Rückstand hatte alle Charaktere der Tartroweinsäure, ohne Spuren von Weinsteinsäure zu enthalten. Nach Verdünnen mit Was̀ser erhielt man daraus durch spontanes Verdunsten Krystalle von Tartroweinsäure.

Die Tartroweinsäure bildet sich also durch die blosse Reaction der reagirenden Elemente, und ist nicht das $f_{\text {Re- }}$ 
sultat der Wirkung einer Base auf die saure Flüssigkeit. Durch die Sättigung mit Baryt bilden sich immer auch weinsteinsaurer Baryt, eir Salz, welches schwerlöslicher und beständiger ist als das Tartrovinat. Statt die Bildung der Tartroweinsäure zu befördern, strebt der Baryt vielmehr sie zu zerstören, indem er sie in Weinsteinsäure und Alkohol zerlegt.

Das wirkliche Vorhandenseyn der Säure in den weinsteinsauren Alkohol nach dem Kochen, beweiset auch, dass, wenn man das festgewordene. Produkt in einer beliebigen Menge Wasser aulöst, dasselbe auf das polarisirte Licht mit einer Energie wirkt, die grösser ist, als die der Weinsteinsäure ursprünglich, so dass also die Moleküle dieser letztern eine Modifikation erleiden mussten durch ihre Verbindung mit dem Alkohol oder seinen Elementen.

\section{¿. 2. \\ Tartrozoeinsäure.}

Man stellt dieselbe dar durch Zersetzen einer Aullösung von tartroweinsaurem Baryt mit Schwefelsäure. Man filtrirt die Flïssigkeit durch ein gereinigtes Filter und verdunstet in der Leere, bis der feste und krystallinische Rückstand keinen Gewichisveriust mehr erleidet.

Die Tartroweinsäure ist schön weiss, geruchlos, von einem zuckrigen, angenehm sauren Geschmack, krystallisirt in zugespitzten Prismen mit schiefer Basis, ist schwerer als Wasser und sehr hygroskopisch.

In Wasser und Alkuhol ist sie leichllöslich, in Aether unlöslich; sie brennt mit einer der des Alkohols ähnlichein Flamme, und riecht dabei wia brennende Weinsteinsäure. Wird sie sechs Stunden lang mit der 4ofachen Menge Wasser gekocht, so zerfällt sie in Alkohol und Weinsteinsäure.

Bei $30^{\circ} \mathrm{C}$. wird sie weich, bei $90^{\circ}$ ist sie in vollem 
Fluss wie ein dïnner Syrup, ihre Flüssigkeit vermehrt sich bis zu $140^{\circ}$, wo sie anfängt, Dämpfe zu entwickeln, die bei $165^{\circ}$ so häufig sind, dass man glauben. möchte, die Flüssigkeit sey im Kochen. Es gehen Alkohol, Wasser, Essigälher, Essigsäure, Kohlensäure und Kohlenwasserstoffgas über und bei $180^{\circ}$ findet man in der Retorte eine Säure, die der sehr gleicht, welche Braconnot erhielt, durch augenblickliches lebhaftes Erhitzen der Weinsteinsäure. Bei $200^{\circ}$ erhielt man ausser den bemerkten Produkten ein flüchtiges Oel und eine dem Brenzessiggeist analoge Flüssigkeit. Als Rückstand bleiben Kohle, Brenzweinsteinsäure und eine ölige Materie.

In einer verdünnten Auflösung der Weinsteinsäure, die der Luft. bei $25^{\circ} \mathrm{C}$. auggesetzt ist, bildet sich Schimmel und in der syrupsartigen Flüssigkeit entstehen Krystalle der unveränderten Säure.

Durch Erwärmen mit Salpetersäure giebt sie Essigsäure, Kohlensäure und 0 xalsäure.

In Schwefelsäure von $66^{\circ}$ löst sie sich ohne. Gasentwicklung. Beim Erhitzen giebt die Auflösung Essigsäure, Kohlensäure, Schweflichtsäure, Kohlenwasserstoff und Spuren von Weinöl.

Zink und Eisen lösen sich in der Säure unter Entwicklung von Wasserstoffgas auf; auf Zinn übt sie weder in der Wärme noch in der Kälte eine Wirkung aus. Wird von einer Auflösung der Säure tropfenweise in Barytwasser gegossen, so entsteht ein Niederschlag, der sich mit Annährung der Neutralität der. Flüssigkeit vermindert; im neutralen Zustande ist die Flüssigkeit noch trübe; fährt man mit dem Zusatz der Säure fort, so erscheint der Niederschlag wieder. Dieses letzte Resultat ist dem Verhalten der Weinsteinsäure ganz. entgegen. In allen Fällen löst Salpetersäure den Nie- 
derschlag auf, aber weil schwerer wenn ein Ueberschuss von Tartroweînsäure vorhanden ist.

Strontianwasser wird durch die Säure in keinem Fall präcipitirt.

Mit Kalkwasser giebt sie einen Niederschlag, der sich im Säureüberschuss wieder aulöst.

In neutralem essigsaurem Blei bewirkt sie kleine in der Säure unlösliche, in Salpetersäure lösliche Prismen, die getrocknet glänzend weiss sind. In concentrirter Lösung von salpetersaurem Silber bewirkt sie einen Niederschlag, der im Säureüberschuss unlöslich ist.

Diese Charaktere lassen die Säure leicht von der Weinsteinsäure unterscheiden.

0,75 Säuren geben durch die Analyse 1,108 Kollensäure und 0,383 Wasser. I Säure lieferte 1,48ı Kohlensäure und 0,521 Wasser.

Dieses giebt für die Zusammensetzung:

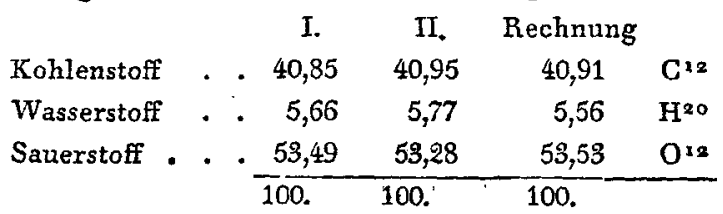

Beide Analysen stimmen überein mit dex rationellen Formel, 2 At. wasserleere Weinsteinsäure, 1 At. Aether und 1 At. Wasser; dieses letzte wird entfernt, wenn man die Säure mit Silberoxyd verbindet.

Ich bernerke, dass dieses die erste Säure dieser Art, die im krystallisirten Zustande analysirt worden ist.

\section{3.}

\section{Tartrovinate.}

Die Tartrovinate sind löslich in Wasser und schwerlöslich in concentrirtem Alkohol, in verdünnten lösen sie sich leicht. Sie krystallisiren im Allgemeinen in ausgezeichneten 
Formen. Sie besitzen ein feltes Anfühlen und brennen mit einer Flamme, die der des Alkobols ähnlich ist.

In der Wärme schmelzen die Alkali -Tartrovinate zwischen $19^{5}$ u. $215^{\circ} \mathrm{C}$., einige Grade stärker erhitzt werden sie zersetzt, unter Entwicklung von Wasser, Alkohol, Essigäther, Essigsäure, wenig fluichtigem Oel, Kohlenwasserstoff und Kohlensäure, unter Hinterlassung von Kohle und Pyrotartrat, wenn die Hitze nicht zu stark war.

Durch langes Kochen in Wasser, werden sie in Alkohol und saure Tartrate verwandelt.

Mit Alkalien bei 160 bis $170^{\circ}$ behandelt, entwickeln sie Alkohol, Essigäther und eine ölige äusserst bittre Materie.

Die Analyse dieser Verbindungen beweist, dass man sie nicht als Salze, worin der Alkohol als Base, betrachten kann.

Die Tartrovinate kann man darstellen mittelst Weinsteinsäure, Alkohol und ein Carbonat, oder durch Doppelzersetzung.

Mit Ausnahme des tartroweinsauren Silbers, welches wasserleer ist, enthalten alle, die ich untersuchte, Krystallwasser, was man ihnen in der trocknen Leere entziehen kann. In den neutralen Salzen verhält sich der Sauerstoff der Säure zu dem der Base, wie 11 zu 1. Krystallwassertrei kann man die betrachten, als bestehend aus 2 At. wasserleerer Weinsteinsäure, 1 At. Aether und a At. Basis. Sie haben folglich eine ganz gleiche Zusammensetzung, wie die von Marchand und Liebig kürzlich studirten Sulfovinate.

Tartroweinsaurer Baryt. Dieses Salz bildet schöne büschelförmig zusammengehäufte Krystalle, deren Krystallsystem das schiefe rhomboidale Prisma ist. Die Winkel liessen sich wegen mangelhafter Ausbildung der Krystalle nicht bestimmen. Zuweilen erbält man es in perlmutterglänzenden 
rhomboidalen Blättchen. Es ist-weiss, gemuchlos, und schmeckt bitterlich.

100 Wasser von $26^{\circ}$ losen 38,12 Salz und 127,64 bei $100^{\circ} \mathrm{C}$. In Alkohol und wasserleerem Holzgeist ist es unlöslich und schwerlöslich in Alkohol von $9^{5^{\circ}}$.

Bei $190^{\circ}$ wird das Salz weich, schmilzt bei $200^{\circ}$ und entwickelt dann einen Alkohol- und Aethergeruch. Die Analyse ergab:

\begin{tabular}{|c|c|c|c|}
\hline & Analyse & Rechnung & \\
\hline Kohlenstoff . & 27,56 & 27,69 & $\mathrm{C}^{12}$ \\
\hline Wasserstoff & 4,22 & 4,14 & $\mathrm{H}^{22}$ \\
\hline Baryt . . & 28,78 & 28,89 & $\mathrm{BaO}$ \\
\hline \multirow[t]{2}{*}{ Sauerstoff } & 39,44 & 39,28 & $0^{13}$ \\
\hline & & 100. & \\
\hline
\end{tabular}

Die rationelle Formel für das Salz ist hiernach: $\mathrm{C}^{8} \mathrm{H}^{8}$ $\mathrm{O}^{\text {to }}, \mathrm{C}^{4} \mathrm{H}^{20} \mathrm{O}, \mathrm{Ba} \mathrm{O}+\mathrm{H}^{4} \mathrm{O}^{2}$, oder: $\mathrm{C}^{4} \mathrm{H}^{4} \mathrm{O}^{5}, \mathrm{BaO}+$ $\mathrm{C}^{4} \mathrm{H}^{4} \mathrm{O}^{5}, \mathrm{C}^{4} \mathrm{H}^{10} \mathrm{O}+\mathrm{H}^{4} \mathrm{O}^{2}$.

In der trocknen Leere verliert das Salz $7,15 \%$ Wasser, was 2 Atomen entspricht.

Für das Aequivalent der Tartroweinsäure resultirt aus der Analyse die Zahl 2130,20 aus der rationellen Formel folgt die Zahl 2129,57 .

Den trocknen tartroweinsauren Baryt kann man ansehen als gebildet aus 2 At. wasserleerer Weinsteinsäure, I At. Aether und 1 At. Baryt. Seine Zusammensetzung entspricht ganz der des schwefelweinsauren Baryts.

Die Tartroweinsäure in dem getrockneten Barytsalze ist endlich :

$\begin{array}{llllrr}\text { Kohlenstoff } & \cdot & \cdot & \cdot & 43,07 & \mathrm{C}^{12} \\ \text { Wasserstoff } & \cdot & \cdot & \cdot & 5,27 & \mathrm{H}^{18} \\ \text { Sauerstoff } & \cdot & \cdot & \cdot & 51,66 & \mathrm{O}^{12}\end{array}$

Die Formel $\mathrm{C}^{12} \mathrm{H}^{18} \mathrm{O}^{\text {rx }}$ kann man auch stellen $\mathrm{C}^{8}$ $\mathrm{H}^{8} \mathrm{O}^{\mathrm{r}}{ }, \mathrm{C}^{4} \mathrm{H}^{\mathrm{r}}{ }^{\circ} \mathrm{O}$, welches ein Bitartrat des Aethers darstellte. 
Tartroweinsaures Kali. Dieses Salz ist weiss, geruchlos, schmeckt kaum bitter, krystallisirt in rhomboidalen Prismen von $124^{\circ}$ und $56^{\circ}$, mit Abstumpfung auf der scharfen Kante von $112^{\circ} 30^{\prime}$. 100 Wasser von $23^{\circ}$ lösen 105,83 Salz und von $100^{\circ}$ jedes Verhältniss. In der Kälte ist es unlöslich in Alkohol von $9^{\circ}$ und in wasserleeren Holzgeist; kochender absoluter Alkohol nimmt nur Spuren davon auf.

Bei $200^{\circ} \mathrm{C}$. wird es weich und fliesst bei $205^{\circ}$.

Die wässrige Auflösung dieses Salzes setzt bei mässiger Wärme und Luftzutritt doppelt weinsteinsaures Kali ab, und es bildet sich Alkohol. Der Niederschlag wird durch langes Kochen vermehrt. Man kann dieses Salz darstellen durch Zersetzen von tartroweinsaurem Baryt mit einem schwachen Ueberschuss von schwefelsaurem Kali, Verdampfen des Filtrats zur Syrupsconsistenz, Auflösen in Alkohol, um den Rückhalt von schwefelsaurem Kali zu entfernen und freiwilliges Verdunsten der fillrirten Flüssigkeit.

Die Analyse dieses Salzes ergab:

\begin{tabular}{|c|c|c|c|c|}
\hline Kohlenstoff & . & $\begin{array}{c}\text { Versuch } \\
. \quad 32,20\end{array}$ & $\begin{array}{c}\text { Rechnung } \\
\text { 32,38 }\end{array}$ & $C^{12}$ \\
\hline Wasserstoff & . & 4,44 & 4,40 & $\mathrm{H}^{20}$ \\
\hline Kali . . & . & 20,78 & 20,83 & $\mathrm{KO}$ \\
\hline Sauerstoff & . & 42,58 & 42,39 & $\mathrm{O}^{22}$ \\
\hline & & 100. & 10 & \\
\hline
\end{tabular}

Die rationelle Formel dafür ist: $\mathrm{C}^{3} \mathrm{H}^{3} \mathrm{O}^{\text {ro }}, \mathrm{C}^{4} \mathrm{H}^{\text {г }}$ $\mathrm{O}, \mathrm{K} \mathrm{O}+\mathrm{H}^{2} \mathrm{O}$. Nach dieser Formel enthält das Salz 3,97 $\frac{\circ}{0}$ Krystallwasser, der Versuch gab $4 \frac{\mathrm{o}}{\sigma}$.

Die Tartroweinsäure gieb $\downarrow$ mit Kali auch ein alkalisch reagirendes Salz, welches in 8 seit. mebrfach zugespitzten Prismen krystallisirt, und deren Disposition eine schiefe Basis anzuzeigen scheint.

Tartroweinsaures Natron. Es ist weiss und krystallisirt in rhomboidalen und rectangulären Blättchen. Es wird wie 
das Kalisalz dargestellt und besteht aus 1 At. Säure, 1 At. Base und $2 \frac{T}{2}$ At. Wasser.

Tartroweinsaurer Kalk. Krystallisirt in reissen, lamellenartigen rectangulären Prismen, geräth bei $130^{\circ} \mathrm{C}$. in wässrigen, bei $210^{\circ}$ in feurigen Fluss, und wird bei $215^{\circ}$ zersetzt. Er wird wie das Barytsalz bereitet und enthält $5 \mathrm{At}$. Krystallwasser.

Anderthalbtartruweinsaures Eupferoxyd. Es ist blau, efflorescirend, krystallisirt in nadelförmigen, seidenglänzenden, verworren gruppirten Prismen. Es wird durch Kupferoxyd und Tartroweinsäure in mässiger Wärme erhalten und enthält 6 Atome Wasser.

Tartroweinsaures Silberoxyd. Es ist weiss, krystallisirt in Prismen, und ist schwerlöslich in Wasser, im Lichte wird es erst röthlich, dann braun. Bei $100^{\circ} \mathrm{C}$. wird es zersetzt, sowohl trocken als in wässrigter Auflösung.

Zur Darstellung giebt man eine concentrirte Auflösung von salpetersaurem Silber in eine concentrirte Auflösung von tariroweinsaurem Baryt oder - Kali, mit Beachtung eines Ueberschusses des letzten. Es schlägt sich eine Menge prismatischer Krystalle nieder. Der Nięderschlag muss mit kaltem Wasser ausgewaschen und unter Ausschluss des Lichis getrocknet werden, in einer Temp. die $50^{\circ}$ nicht überschreitet. Er hält dann kein Wasser mehr zurück. Die Analyse ergab :

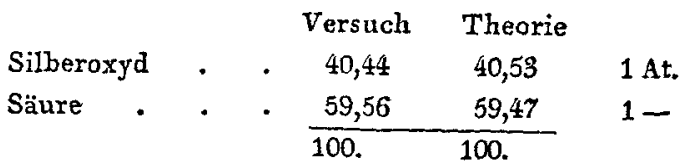

Das Mischungsgewicht der Säure ist hiernach 2137,91 . Tartroweinsaures Ammoniak. Es wird dargestellt durch genaues Sälligen der Säure mit kohlensaurem Ammoniak, und freiwilliges Verdunsten der Auflösung. Es bildet feine seidenglänzende rhomboidale Prismen. 
4. 4.

\section{Wirkung der Paraveinsteinsäure auf den Alkohol.}

Die Bezichung der Paraweinsteinsäurer zur Weinsteinsäure machten es interessant, auch die Wirkung erster auf denAlkohol zu erforschen. Ich wand te reine schöne Krystalle an. Die Krystalle sind schiefe Prismen, und grosse durchscheinende Rhomben. Der Winkel zweier Flächen ist $110^{\circ}$, die Neigung der Basis auf eine dieser Flächen ist $106^{\circ} 30^{\prime}$ und auf die andere Fläche $129^{\circ}$. Die Krystallform ist in Bezug auf die Winkel wenigstens sehr verschieden von der der Weinsteinsäure.

$\mathrm{Da}$ die Paraweinsteinsäure in Alkohol schwerlöslicher ist als die Weinsteinsäure, so muss man wenigstens $4 \mathrm{Th}$. Alkobol anwenden auf einen Theil Säure. Man hört zu erhitzen auf, wenn die Flüssigkeit bei Syrupsconsistenz nach Erkalten nichts mehr absetzen lässt, man verdünnt sie mit Wasser, sättigt mit kohlensaurem Baryt, und nach Verdampfen bei 50 bis $60^{\circ}$ setzt man sie der Luft aus und behandelt weiter wie oben bei der Weinsteinsäıre angeführt worden ist.

\section{Paratartroweinsäure.}

Diese Säure ist weiss, geruchlos, schmeckt elwas mehr zuckerig als die Tartroweinsäure, krystallisirt in dieser ähnlichen Prismen, ausgenommen dass die Base geneigter ist gegen die Achse bei der Tartroweinsäure als bei der Paratartroweinsäure.

Sie hat keine merkliche Wirkung auf das polarisirte Licht, nicht mehr als die Paratartroweinsäure. Gegen Wasser, Alkohol, Aether und beim Erhitzen, gegen Schwefelsäure, Salpetersäure, Zink, Eisen und Zinn, so wie gegen Ḅarytwasser verhält sie sich wie die Tartroweinsäure. Mit ihrem vierzigfachen Gewicht Wasser gekocht, zersetzt sie sich in Alkohol und Paraweinsteinsänre, die krystallisirt. 
In Kalkwasser bringt sie einen im Ueberschuss der Säure unlöslichen, in Salpetersäure auflöslichen Niederschlag hervor. In Strontianwasser giebt sie einen im Ueberschuss der Säure löslichen Niederschlag, mit Kali einen pulverförmigen Niederschlag, wenn die Säure vorherrscht. In Natronlösung bewirkt sie einen opalinischen Niederschlag etwas vor dem Neutralwerden der Flïssigkeit, der sich mit der Menge der Säure vermehrt, und in kaltem Wasser unlüslich ist; eben so verhält sie sich gegen kohlerisaures Natron. ' In schwefelsaurem Kalk und schwefelsaurem Natron bringt sie auch nach 24 Stunden keinen Niederschlag herwor.

Essigsaures Blei und salpetersaures Silber, dieses in concentrirter Lösung, schlägt sie nieder, der Niederschlag aus letzter bildet dem tartroweinsauren Silber ähnliche Prismen.

Die Analyse der Säure ergab:

\begin{tabular}{|c|c|c|c|c|c|}
\hline \multirow[b]{2}{*}{ Kohlenstoff } & & \multicolumn{2}{|c|}{ Versuch } & \multicolumn{2}{|l|}{ Rechnung } \\
\hline & . & 38,77 & 33,54 & 38,95 & $C^{22}$ \\
\hline Wasserstoff & . & $5,9 \cdot 1$ & 5,94 & 5,83 & $\mathrm{H}^{22}$ \\
\hline Sauerstoff & • & 55,32 & 55,52 & 55,22 & Oxs \\
\hline & & 100. & 200. & 100. & \\
\hline
\end{tabular}

Die gemeine Formel $\mathrm{C}^{\mathrm{rr}} \mathrm{H}^{22} \mathrm{O}^{3}$ lässt sich in die rationelle verwandein $\mathrm{C}^{8} \mathrm{H}^{8} \mathrm{O}^{\text {ro }}, \mathrm{C}^{4} \mathrm{H}^{\text {ro }} \mathrm{O}+\mathrm{H}^{4} \mathrm{O}^{2}$, welches 2 Atome Paraweinsteinsäure, I Atom Aether und 2 At. Wasser giebt. Sie enthält also I Atom Wasser mehr als die Tartroweinsäure. Sie gleicht hierin der Paraweinsteinsäure, die auch 2 Atome Wasser enthält, während die Weinsteinsäure nur I At. enthält.

\section{Paratartrovinate.}

Von diesen gilt mit einigen Ausnahmen dasselbe, was von den Tartrovinaten gilt. Sie geben z. B. nicht so schöne Krystalle, und einige schliessen andere Verhältnisse von Krystallwasser ein als die entsprechenden Tartrovinate.

Paratartroweinsaurer Baryt. Dieser krystallisirt in 
kleinen warzenförmig zusammengehäuften Prismen, und unterscheidet sich dadurch wesentlich vom tartroweinsauren Baryt. Er ist weit löslicher im warmen als im kalten Wasser; in Alkohol von $9^{5} \frac{\circ}{0}$ und in wasserleerem Holzgeist ist er unlöslich. Die Analyse ergab:

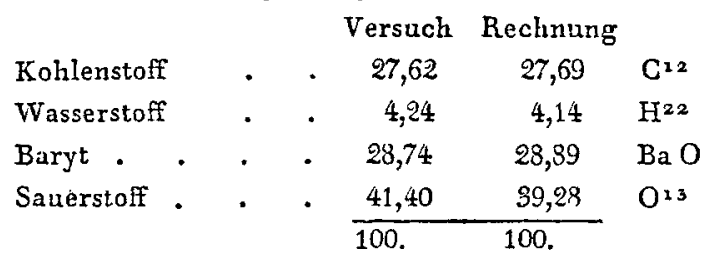

Die raticnelle formel ist $\mathrm{C}^{8} \mathrm{H}^{8} \mathrm{O}^{10}, \mathrm{C}^{4} \mathrm{H}^{\times 0} \mathrm{O}, \mathrm{BaO}+$ $\mathrm{H}^{4} \mathrm{O}^{2}$. Dieses giebt 6,80 응 Krystallwasser, der Versuch gab 6,95 .

Die Zusammensetzung dieses Salzes ist dieselbe wie die des tartroweinsauren Baryts und die Paralartroweinsäure ist isomerisch mit der Tartroweinsäure.

Paratartroweinsaures Käli. Dieses ist weiss, schmeckt wie tartroweinsaures Kali, krystallisirt aber nicht so gut. Die Krystalle scheinen Prismen mit vierseitiger Basis zu seyn, deren Grundkanten mit sehr schiefen Flächen abgestumpft sind. Es besteht aus :

$$
\begin{array}{lccccc} 
& & & & \text { Versuch } & \text { Rechnung } \\
\text { Kali } & \cdot & \cdot & \cdot & 19,95 & 20,03 \\
\text { Säure } & \cdot & \cdot & \cdot & 72,40 & 72,34 \\
\text { Wasser } & \cdot & \cdot & \cdot & 7,65 & 7,63 \\
\hline & & & & 100 . & 100 .
\end{array}
$$

Paratartroweinsaures Silberoxyd. Es verhält sich ganz wie das Tartrovinat. 2 Gram. gaben 0,754 Gram. metallisches Silber, folglich 40,5 Silberoxyd für 100 Salz.

Das Atomengewicht der Paratariroweinsäure ist hiernach $213 z, 6$ statt 2129,57 , aus der Formel für das Barytsalz abgeleitet. Die Zusammensetzung ist dieselbe wie beim tartroweinsaurem Silberoxyde 


\section{Zweiter Theil. \\ Wirkung der Weinsteinsäure und Paraweinsteinsäure auf den Holzgeist.}

Die von $\mathrm{Dum}$ as und Peligot nachgewiesene Analogie zwischen Holzgeist und Alkolıol bewog mich, die vorstehenden Versuche mit Holzgeist zu wiederholen. Da beide Säuren in dieser Flüssigkeit löslicher sind als in Alkohol, so wird der Holzgeist eher ätherificirt als letzter.

\section{Tartromethylsäure.}

Man kann sie eben so wie die Tartroweinsäure bereiten, es ist aber einfacher, nach Aullösen von 1 Theil Weinsäure in $1 \mathrm{Th}$. wasserleeren oder wasserhalligen Holzgeist durch Wärme, die Flïssigkeit bei einer Temp. unter $100^{\circ} \mathrm{C}$. zur Syrupsdicke einzuengen. Wenr die Destillation langsamer geht, versucht man, ob der Syrup keine Weinsteinsäure mehr entbält, enthälf er noch davon, so kohobirt man, oder setzt die Operation fort. Im Gegentheil löst man die Masse in der Hälfte ihres Gewichts Wasser, verdampft unter $100^{\circ} \mathrm{C}$, , und ïberlässt die sehr dicke Flüssigkeit der freiwilligen Verdunstung. Es setzen sich Krystalle von Tartromethylsäure ab, meistens aber gesteht das Ganze zu einer krystallinischen Masse, die man in der Leere trockrret.

Die Auflösung der syrupsertigen Masse und Verdunstung lat zum Zweck, den Holzgeist gänzlich zu verjagen, woron sonst immer ein Rï̈ckhalt bleibt.

Die Tartromethylsäure ist weiss, geruchlos, von saurem Geschmack, nicht sïss wie die Tartroweinsäure, dichter als Wasser, krystallisirt in Prismen, ist hygroskopisch, leichtlöslich in Wasser, auch in Alkohol und Holzgeist, schwerlöslich in Aether, und brennt mit einer der des 
Holzgeistes ähnlichen Flamme. Durch Kochen mit Wasser wandelt sie sich in Holzgeist und Weinsteinsäure um, doch etwas schwerer als Tartroweinsäure.

In der Hitze wird sie flïssig, giebt dann Wasser, Holzgeist, Methylenacetat und eine sehr dichte Flüssigkeit, in welcher ich die Gegenwart des Methylenoxalates, dessen Bildung mir unter diesen Umständen möglich schien, nicht nachweisen konnte.

Wird eine Auflösung der Methylsäure der freiwilligen Verdunstung überlassen, so bleibt eine Masse von Krystallen zurück, die dieselbe Eigenschaft haben, wie die Süure vor ihrer Auflösung.

Gegen Eisen, Zink und Zinn verhält sie sich wie die Tartroweinsäure. Mit Baryt-, Kalk- und Strontianwasser bildet sie im Ueberschuss der Säure Iösliche Niederschläge.

In Kalilösung giebt sie einen Niederschlag, wenn die Flüssigkeit wenig sauer ist. Er ist nicht körnig wie bei der Weinsteinsäure, und erscheint auch unler dem Mikroskop nicht krystallinisch. Auch mit Natron giebt sie einen reichlichen Niederschlag, so bald die Flüssigkeit sauer ist, er ist körnig aber nicht krystallisirt, unlöslich in einem Säureüberschuss, aber löslich in vielem Wasser.

In schwefelsaurem Kali und - Natron bringt sie keinen Niederschlag hervor; im essigsaurem Blei bewirkt sie einen. anfangs flockigen, nachher bei Säureüberschuss pulverigten Niederschlag, in concentrirter salpetersaurer Silberlösung einen flockigen in Ueberschuss der Säure unlöslichen Niederschlag, der in Wasser ein wenig löslich ist. Ihre Zusammensetzung ist :

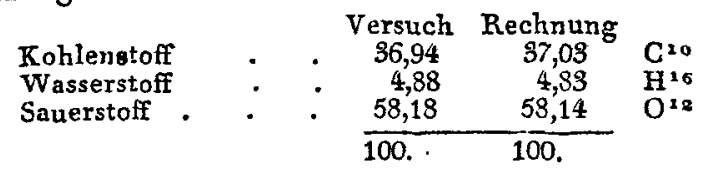

Dieses giebt die rationelle Formel: $\mathrm{C}^{3} \mathrm{H}^{8} \mathrm{O}^{\mathrm{x}}, \mathrm{C}^{2} \mathrm{H}^{6}$ 
$\mathrm{O}+\mathrm{H}^{2} \mathrm{O}, 2$ At. Weinsteinsüure, 2 At. Methylenmonobydrat, und 1 At. Wasser.

\section{Tartromethylsaure Salze.}

Tartrometkylsaures Kali. = Man erhält es wie das Tartrovinat. Es ist weiss, 'geruchlos, krystallisirt in graden rectangulären Prismen, ist in warmen Wasser leichtlöslicher als in kaltem, unlöslich in wasserleerem Holzgeist und Alkohol von $95 \%$.

Bei $150^{\circ} \mathrm{C}$. wird es weich und gelblich, bei $130^{\circ}$ entwickelt es weisse Dämpfe, bei $200^{\circ}$ ist die Zersetzung in voller Thätigkeit, es entwickelt sich Kohlenwasserstoff, Kohlensäure, Melbylenacelat, Holzgeist, Essigsäure, Wasser und eine" dickliche Materie; durch anlialtendes Kochen mit Wasser wird es zu Holzgeist und doppeltweinsteinsaurem Kali. Die Zusammensetzung ist:

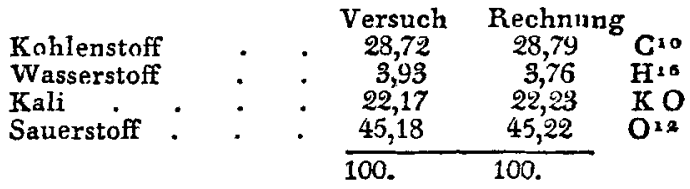

Hieraus ergiebt sich die rationelle Formel: $\mathrm{C}^{8} \mathrm{H}^{8} \mathrm{O}^{\mathrm{ro}}$, $\mathrm{C}^{2} \mathrm{H}^{6} \mathrm{O}, \mathrm{KO}+\mathrm{H}^{2} \mathrm{O}$. Darnach enthält das Salz $4,23 \frac{\circ}{0}$ Krystallwasser, statt 4,20 nach dem Versuch. Das Atomengewicht der Tartromethylsäure ist nach dieser Formel $19^{51,73}$, was nahe mit der obigen, 1959,2 zusammentrifft.

Tartromethylsaurer Baryt. Dieses Salz ist von D u mas und Peligot analysirt worden. Sie fanden darin 1 At. Tartromethylsäure, 1 At. Baryt und 1 At. Wasser. Sie erhielten dasselbe durch Vermischen einer Aufösung von Baryt in Holzgeist mit Weinsteinsäure, ebenfalls in Holzgeist gelöst, und Auswaschen des Niederschlages mit wasserleerem Holzgeist.

Arch. d.Pharm. II. Reihe. IX.Bda. 3. Hft. 
Das Salz, welches ich untersuchte, wurde wie das Tartrovinat dargestellt, da es aber schwieriger krystallisirt, so wurde die Auflösung der freien Verdunstung übèrlassen; auch in gelinder Wärme erhält man' oft nur eine syrupsartige Masse ohne Krystalle.

Der tartromethylsaure Baryt ist weiss, von bitterm Geschmack, krystallisirt in geraden mitunter zugespitzten Prismen; ist unlöslich in wasserleerem Holzgeist und Alkohol von $9^{\circ}$, löslicher in warmen als in kaltem Wasser, und zersetzt sich beim Kochen darin leichter als das Kalisalz.

Bei 150 bis $160^{\circ} \mathrm{C}$. zersetzt er sich zu einer knoblauchartig riechenden dicken Flüssigkeit, die Holzgeist, Wasser, Methylenacetat und eine krystallisirte Substanz enthält, die leichtlöslich in Wasser, aber kein Methylenoxalat ist.

\section{Paratartromethylsäure.}

Sie wird wie die Tartroweinsäure, oder besser nach dem bei der Tartromethylsäure angegebẻnen zweiten Verfahren bereitet.

Sie ist weiss, geruchlos, schmeckt wie die Tartromethylsäure, krystallisirt in geraden rectangulären, an den Seitenkanten abgestumpften Prismen, die in rhombische Prismen übergehen; verhält sich gegen $W$ asser, Alkohol und Aether in der Hitze, gegen Eisen, Zink und Zinn wie die Tartromelhylsäure. Ihre wässrigte Auflösung wird nicht durch freiwilliges Verdunsten an der Luft, aber durch Kochen zersetzt in Holzgeist und Paraweinsteinsäure.

In Barytwasser bringt sie einen im Ueberschuss der Säure und in Wasser löslichen Niederschlag hervor.

In Strontianwasser bewirkt sie einen in Säureüberschuss unlöslichen Niederschlag, der sich aber in viel Wasser auflöst.

Mit Kalkwasser giebt sie einen aus nadelförmigen Pris- 
men zusammengehäuften Niederschlag, der in Säureüberschuss unlöslich ist. Mit Natron und kohlensaurem Natron giebt sie in keinem Fall einen Niederschlag, diese drei letzten Charaktere unterscheiden sie von der Tariromethylsäure.

Mit Kali giebt sie, wenn die Flïssigkeit sauer ist, einen Niederschlag, der in vielem Wasser löslich ist. In neutralem und basisch essigsaurem Blei bringt sie einen in Säureüberschuss unlöslichen Niederschlag hervor, in schwefelsaurem Kali giebt sie keinen Niederschlag, in concentrirter Auflösung von salpetersaurem Silber bewirkt sie weisse, in Säureüberschuss unlösliche Flocken.

Ihre Zusammensetzung ist :

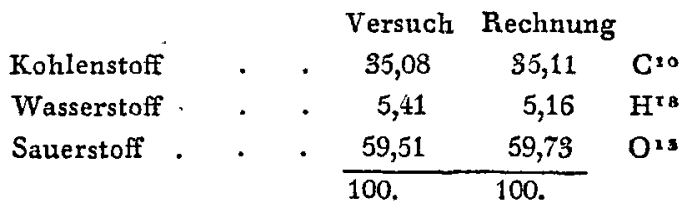

Hieraus resultirt die rationelle Formel: $\mathrm{C}^{8} \mathrm{H}^{8} \mathrm{O}^{\mathrm{ro}}, \mathrm{C}^{2}$ $\mathrm{H}^{6} \mathrm{O}+\mathrm{H}^{4} \mathrm{O}^{2}$.

Die Zusammensetzung der Paratartromethylsäure ist der der Paratartroweinsäure also ganz analog.

\section{Paratartromethylsaure Salze.}

Paratartromethylsaures Kali. Es wird wie das tartroweinsaure Kali bereitet. Es ist weiss, geruchlos, krystallisirt in geraden Prismen, ist in warmen Wasser löslicher als in kaltem, unloslich in Holzgeist und Alkohol von $9^{5} \frac{\circ}{\circ}$. Bei $100^{\circ} \mathrm{C}$. wird es weich, bei $150^{\circ}$ fängt es an zersetzt zu werden und giebt bei $200^{\circ}$ dieselben Produkte wie das Tartromethylat. Durch langes Kochen in Wasser wird es zu Holzgeist und doppelweinsteinsaurem Kali.

Seine Zusammensetzung ist: 


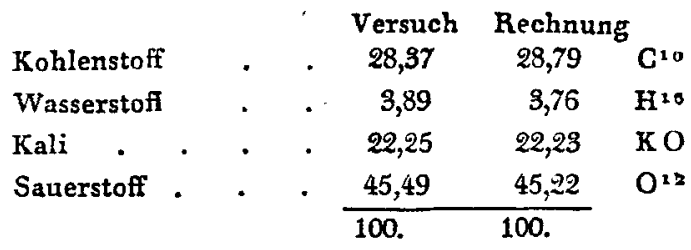

Die rationelle Formel dieses Salzes ist: $\mathrm{C}^{8} \mathrm{H}^{8} \mathrm{O}^{\mathrm{O}}, \mathrm{C}^{2}$ $\mathrm{H}^{6} \mathrm{O}, \mathrm{KO}+\mathrm{H}^{2} \mathrm{O}$, also dieselbe Zusammensetzung wie das Tartromethylat. 100 Salz enthalten nach der Formel 4,23 Krystallwasser, nach dem Versuch 4,25. Das Atomengewicht der Säure ist 1915,73 , gefunden wurde 1948,71 .

Paramethylsaurer Baryt. $\mathrm{Er}$ ist weiss, schmeckt wie tartromethylsaurer Baryt, krystallisirt in Prismen mit parallelogrammischer Grundfäche; enthält 4 Atome Krystallwasser, verliert davon 3 an der Luft, indem es opak wird.

Das nicht efflorescirte Salz wird bei $60^{\circ}$ weich, giebt bei $100^{\circ} \mathrm{C}$. Dämpfe aus, die sich zu krystallinischen Blättchen verdichten, schmelzt bei $105^{\circ}$, kömmt bei $120^{\circ}$ ins Kochen, wird bei $17^{\circ}$ gelblich und bei $205^{\circ}$ zersetzt. Es giebt dabei eine Flüssigkeit, bestehend aus Wasser, Methylenacetat, Holzgeist und eine krystallinische Substanz, die man durch langsames Verdunsten erhält, und der bei $100^{\circ}$ erhaltenen gleich zu seyn scheint. Das nicht efflorescirte Salz entwikkelt diese Substanz erst bei $130-140^{\circ}$. Sie ist kein Methylenoxalat, wie man glauben möchte, vielleicht ein Methylentartrat, und bedarf noch einer genauern Untersuchung.

Der paratartromethylsaure Baryt ist weit leichtlöslicher in warmen als in kaltem, Wasser, unlöslich in wasserleerem Holzgeist und in Alkohol von $9^{5} \frac{\circ}{0}$. Die Zusammensetzung ist :

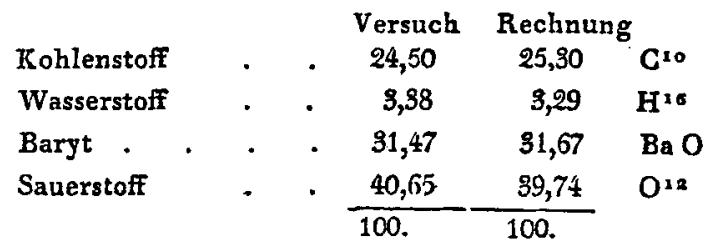


Hieraus ergiebt sich die rationelle Formel: $\mathrm{C}^{8} \mathrm{H}^{8} \mathrm{O}^{ \pm 0}$, $\mathrm{Ca}^{6} \mathrm{O}, \mathrm{BaO}+\mathrm{H}^{2} \mathrm{O}$. 100 Salz enthalten hiernach 3,72 Krystallwasser, der Versuch ergah 3,80. Die Zusammensetzung dieses Salzes ist dem Tartromethylat ganz analog.

Bemerkung. Man nimmt im Allgemeinen an, dass bei der Analyse eines Alkalisalzes mit organischer Säure ein basisches Carbonat entstehe, was einen Irrthum in der Bestimmung des Kohlensloffs herbeiführt, bei den Alkalisalzen, die ich analysirt habe, habe ich gefunden, dass wenn ich eine Quantität Kupferoxyd anwandte; die das 184fache Gewicht der organischen Materie betrug, niemals ein Carbonat in der Verbrennungsröhre zurückblieb.

\section{Schlïsse.}

Die Weinsteinsäure und Paraweinsteinsäure verwandeln den Alkohol in Tartro - und Paratartroweinsäure, und den Holzgeist in Tartromethyl - und Paratartromethylsäure. Diese Umwandlung findet in der Wä́rme sogleich statt, und in gewöhnlicher Temp. nach einiger Zeit.

Es ist kein so grosser Unterschied, als man gewöhnlich glaubt, zwischen der ätherbildenden Kraft der Weinstein und Paraweinsteinsäure gegen die der Schwefelsäure.

Da die Weinstein - und Paraweinsteinsäure die Umwandlung des Alkohol - und Holzgeistes in Aether bewirken, ohne Zwischenkunft der Schwefelsäure, so glaube ich, dass dieses auch alle, selbst die schwächsten Säuren vermögen, wenn sie in Alkohol oder in Holzgeist löslich sind.

Die krystallisirte Tartroweinsäure kann vorgestellt werden durch 2 At. wasserfreie Weinsteinsäure, 1 At. Aether und 1 At. Wasser oder als Bitartrat von Aether-Monohydrat. Die Paratartroweinsäure hat dieselbe Sättigungscapacität wie lie Tartroweinsäure; sie enthält im krystallisirten Zustande nur I Atom Wasser mehr. Es ist bemerkenswerth, dass, 
wie bei der Paraweinsteinsäure, der blosse Ueberschuss von einem Atome Wasser dem Produkte alle merkliche Wirkung auf das polarisirte Licht nimmt.

Die Tartrovinate und Paratartrovinate, welche Krystall_ wasser enthalten, verlieren dasselbe in der trocknen Leere. In den getrockneten Salzen entspricht jede der Säuren zwei Atomen wasserleerer Weinsteinsäure und einem Atome Aether. Sie unterscheiden sich unter diesem Gesichtspunkte von den entsprechenden Sulfovinaten nur dadurch, dass in diesen die Weinsteinsäure durch Schwefelsäure ersetzt ist.

Die Tartromethyl - und Paratartromethylsäure entsprechen völlig der Tartro- und Paratartroweinsteinsäure, eben so die Salze dieser Säuren.

Diese Resultate bestätigen endlich auch die von $\mathrm{Du}$ mas und Peligot festgesetzte Aralogie zwischen Holzgeist und Alkohol.

Beobachtungen über blaue Milch;

von

Henri Braconnot.

(Journ, de Chim. med. 2 Ser. II.T. 625. Auszug.)

Ueber die Natur der blauen Farbe, welche die Milch unter einigen Umständen zeigt, ist man noch nicht einig. Durch Herrn Dr. Lamouroux erhielt ich eine kleine Quantität eines solchen blauen Rahms. Die blaue Farbe ist fast so intensiv wie Ultramin.

In Bezug auf Robiquet's Ansicht, dass die blaue Farbe, vom phosphorsauren Eisenoxydul herrühren möchte, verbrannte ich etwas des blauen Rahms, und erhielt eine weisse Asche, die Eisen enthielt, ohne Zweifel an Phosphorsäure 